\title{
Generalizable design parameters for soluble $T$ cell receptor-based $T$ cell engagers
}

Karen J Froning, Arlene Sereno, Flora Huang, Stephen J Demarest

To cite: Froning KJ, Sereno A, Huang F, et al. Generalizable design parameters for soluble T cell receptor-based T cell engagers. Journal for ImmunoTherapy of Cancer 2022;10:e004281. doi:10.1136/ jitc-2021-004281

- Additional supplemental material is published online only. To view, please visit the journal online (http://dx.doi.org/10. 1136/jitc-2021-004281).

KJF and AS contributed equally. Accepted 02 February 2022

Check for updates

(c) Author(s) (or their employer(s)) 2022. Re-use permitted under CC BY-NC. No commercial re-use. See rights and permissions. Published by BMJ.

Lilly Biotechnology Center, Eli Lilly and Company, San Diego, California, USA

\section{Correspondence to}

Dr Stephen J Demarest, Tentarix Biotherapeutics, San Diego, CA, USA; sdemarest@tentarix.com

\begin{abstract}
While most biological and cellular immunotherapies recognize extracellular targets, T cell receptor (TCR) therapeutics are unique in their ability to recognize the much larger pool of intracellular antigens found on virus-infected or cancerous cells. Recombinant T cell receptor (rTCR)-based therapeutics are gaining momentum both preclinically and clinically highlighted by recent positive phase III human clinical trial results for a TCR/CD3 bifunctional protein in uveal melanoma. Unlike antibody-based T cell engagers whose molecular formats have been widely and extensively evaluated, little data exist describing the putative activities of varied bifunctional formats using rTCRs. Here we generate rTCR/anti-CD3 bifunctionals directed toward NY-ESO-1 or MAGE-A3 with a variety of molecular formats. We show that inducing strong redirected lysis activity against tumors displaying either NY-ESO-1 or MAGE-A3 is highly restricted to small, tandem binding formats with an rTCR/antiCD3 Fab demonstrating the highest potency, rTCR/anti-CD3 single chain variable domain fragment showing similar but consistently weaker potency, and lgG-like or lgG-Fccontaining molecules demonstrating poor activity. We believe this is a universal trait of rTCR bifunctionals, given the canonical TCR/human leukocyte antigen structural paradigm.
\end{abstract}

\section{BACKGROUND}

There are two major categories of cancer immunotherapy. The first involves stimulating disease-targeting immune cells to overcome tumor immunosuppression using activating cytokines or small molecules, immune cell costimulators, or by releasing immune suppression via inhibition of immune checkpoint receptors. ${ }^{1}$ The second involves cellbased or soluble recombinant agents that redirect immune cells toward diseased cells expressing tumor-specific antigens (TSAs). ${ }^{2}$ Targeting can be mediated by transduction of an antibody on autologous or allogeneic immune cells ex vivo for in vivo patient delivery $^{3}$ or via the direct administration of soluble multispecific agents that act in trans to bridge effector immune cells to bind and kill tumor cells. ${ }^{2}$ Both categories are transforming cancer therapy, and combinations of these two categories are an emerging next step in cancer treatment.
TSAs commonly targeted by antibody or antibody-like immunotherapies include cell surface markers expressed on diseased hematopoietic cancer cells or cell surface markers overexpressed via gene amplified, epigenetic upregulation or other mechanisms on solid tumor tissues. ${ }^{2}$ The number of these extracellular targets is limited and their specificity is rarely exclusive to tumor cells. It is estimated that $>70 \%$ of tumor-specific targets consist of intracellular oncogenes, mutated tumorsuppressor proteins, or fusion proteins inaccessible to antibody targeting including CAR T cells, antibody-drug conjugates, and bispecific $\mathrm{T}$ cell engagers. ${ }^{4}$ These intracellular target proteins are accessible to the human immune system through adaptive immune recognition by $\mathrm{T}$ cells. In particular, $\alpha / \beta \mathrm{T}$ cells express $\alpha / \beta$ T cell receptors (TCRs) with the capacity for antibody-like $\mathrm{V} / \mathrm{J}(\alpha)$ and $V / D / J(\beta)$ recombination to generate vast diversity to recognize foreign proteins. CD8+ T cells use their $\alpha / \beta$ TCRs to recognize non-self-peptides complexed and displayed by major histocompatibility complex (MHC) class I proteins that are ubiquitously expressed on somatic cell surfaces. Methods for identifying, sequencing, and functionally verifying $\alpha / \beta$ TCRs have advanced significantly with the discovery of $\alpha / \beta$ TCRs against many of the most common intracellular cancer antigens displayed by the most prevalent human leukocyte antigens (HLAs). ${ }^{5}$

Recombinant T-cell receptor (rTCR)based targeting of tumor cells is gaining momentum as a cancer immunotherapy. ${ }^{6}$ This includes (1) the recombinant transduction of an oncogene-specific or cancer mutation-specific $\alpha / \beta$ TCR into autologous patient $\mathrm{T}$ cells and reintroduction of these $\mathrm{T}$ cells back into patients with the proper $\mathrm{T}$ cell antigen-HLA match or (2) the generation of soluble TCR proteins fused to an antiCD3 single chain variable domain fragment $(\mathrm{scFv})$ to redirect a patient's $\mathrm{T}$ cells to target 
antigen/HLA complexes on tumor cells. ${ }^{6} 7$ The first generation of soluble rTCR/anti-CD3 fusion molecules has been dubbed 'immune mobilizing monoclonal TCRs against cancer' (ImmTACs). While rTCR-T cell therapies have been more prevalent than ImmTACs, these cell therapies suffer from a variety of issues including competition with endogenous TCRs on T cells for the CD3 signaling components, insufficient rTCR expression, insufficient potency, and heterogeneous mixing of the rTCR $\alpha$ and $\beta$ chains with their endogenous counterparts, which could lead to uncontrolled specificity issues and safety concerns, and challenges with engraftment. ${ }^{48}$ Once cell longevity is obtained, dosing and potential long-term toxicity can also be difficult to control, which has spurred the recent development of switches and control methodologies for cell therapies. ${ }^{9}$

Soluble rTCR-based protein therapeutics have not been as prevalent for many reasons but are gaining traction, given advances in the design of antibodybased T cell engagers, ${ }^{2}{ }^{10}$ improved TCR discovery and engineering, ${ }^{7}$ and improved methods for their production. ${ }^{11}$ Importantly, recent positive clinical results from a phase III study of a gp100/HLA-A2-targeted ImmTAC $^{12}$ are the first large-scale clinical trials demonstrating activity for $\mathrm{T}$ cell-engaging bifunctionals and highlight the benefits of rTCR-based therapies. Spearheaded by ImmunoCore, the ImmTAC technology uses affinity matured rTCRs with high potency to enable $\mathrm{T}$ cell redirection toward tumor cells displaying HLA/ peptide complexes with receptor densities as low as 50-100 receptors per cell. ${ }^{7}$

Engineering these molecules for therapy is challenging, given the many parameters required for activity including exquisite specificity and manufacturability. rTCR proteins are generally manufactured in Escherichia coli and require solubilization of protein fragments followed by oxidative folding and chain pairing, typically resulting in low yields. These challenges have hindered the ability to characterize rTCR/anti-CD3 molecules with geometries and valency beyond the ImmTAC format. ${ }^{13}$ Molecular geometry and epitope play a critical role in the activity of bispecific antibody (BsAb)-based $\mathrm{T}$ cell engagers ${ }^{10}$; thus, a greater understanding of the role molecular design plays on the function of rTCR-based T cell engagers is warranted. Unlike BsAbs whose epitopes are highly diverse, TCRs use a canonical epitope/binding mechanism to engage HLA-peptides. Thus, we believe molecular parameters derived for rTCRbased bifunctional proteins (BFPs) that drive their potency and activity will be universal across this class of molecules. Recently, we have described a methodology to express soluble TCRs at antibody-like levels in mammalian cells, ${ }^{11}$ opening the door to different rTCR/anti-CD3 BFP architectures. Here, we explore many rTCR/anti-CD3 BFP geometries and contrast them for their ability to redirect $\mathrm{T}$ cells to kill tumor cells displaying either MAGE-A3/HLA-A1 or NY-ESO-1/HLA-A2 on their cell surfaces. We uncover strict geometrical requirements for high potency and activity of these molecules with rules common to both sets of antigen/
HLAs. These studies lay a general foundation for the design of rTCR/anti-CD3 BFPs.

\section{MATERIALS AND METHODS Production of rTCR/anti-CD3 BFPs}

The NY-ESO- 1 and MAGE-A3 $\alpha / \beta$ TCR as well as antiCD3 SP34 amino acid sequences have been published. ${ }^{11} 14$ DNA GeneBlock up to 900 nucleotides encoding for the rTCR/anti-CD3 BFPs were designed and synthesized by Integrated DNA Technologies. Gene segments with 15 nucleotide $5^{\prime}$ and $3^{\prime}$ overlaps were designed for In-Fusion (Takara Bio USA) cloning of multiple segments into a cytomegalovirus (CMV) promotor-driven mammalian expression vector using HindIII and EcoRI or BamHI and EcoRI restriction sites (Lonza). Briefly, In-Fusion reactions were transformed into E. coli strain Top 10 competent cells (Life Technologies). Colonies were picked and clonal DNA was produced by miniprepping (according to Qiagen MiniPrep cat\#27104 protocol procedures) and sequenced by an in-house DNA sequencing core. Medium-scale and large-scale plasmid purifications were performed according to the instructions within the Plasmid Plus Midi Kit (Qiagen cat\#12945) and Plasmid Plus Maxi Kit (Qiagen cat\#12965), respectively. TCR $\alpha / \beta$ constant domains contained stabilizing designs. ${ }^{11}$ All TCR-scFv, TCR-Fab (antibody antigen binding fragment), and 2TCR-Fab sequences contained an $8 \times$ Histag for affinity purification. IgG1-Fc-containing constructs used an Fc-heterodimerization design (7.8.60) to enable proper assembly ${ }^{11}$ and L234A, L235A, and N297Q mutations to eliminate CH2-domain N-linked glycosylation and any potential effector function. Mature sequences for every construct described in the manuscript are provided in the online supplemental materials.

Transient transfections and protein expressions in Chinese hamster ovary $(\mathrm{CHO})$ cells $(\mathrm{CHO} \mathrm{K} 1 \mathrm{SV})$ were performed as described previously. ${ }^{15}$ Briefly, plasmids were transfected into CHO cells using $8 \mathrm{mg}$ / L Polyethylenimine Max (PEI Max) from Polysciences (cat\#2 47646-2) per $1.5 \mathrm{e} 6$ cells $/ \mathrm{mL}$. DNA for transfection was mixed at $1.6 \mathrm{mg} / \mathrm{L}$ coding plasmid $(\mathrm{s})+1.6 \mathrm{mg} / \mathrm{L}$ herring sperm DNA. Cells were maintained in Dulbecco's Modified Eagle Medium (DMEM)-containing media with $10 \mathrm{mM}$ L-glutamine (LM-Growth, SAFC cat\#59202C-100) and shaken in flasks at $37^{\circ} \mathrm{C}, 6 \%-8 \% \mathrm{CO}_{2}$ in a humidified incubator prior to transfection and at $32^{\circ} \mathrm{C}$ post-transfection. Shaker speed was $250 \mathrm{rpm}$ on a $3 / 4$-inch diameter shaker. Protein secretion into the media was achieved by N-terminal fusion of each sequence to a mouse antibody kappa light-chain signal sequence. Supernatants were collected for protein purification by centrifugal pelleting of the cell mass followed by $0.2 \mu \mathrm{M}$ filtration.

Protein purification was performed using either a onestep or two-step process to achieve $>90 \%$ pure material based on analytical size exclusion. Histagged proteins were affinity purified using a $\mathrm{Ni}^{2+}$-nitrilotriacetic acid (NTA) immobilized resin (cOmplete His-Tag, Millipore 
Sigma) and an AKTA Pure system (GE Healthcare). The column was conditioned in binding buffer $(20 \mathrm{mM}$ Tris$\mathrm{HCl} \mathrm{pH} \mathrm{8.0,0.5} \mathrm{M} \mathrm{NaCl)} \mathrm{prior} \mathrm{to} \mathrm{running} \mathrm{the} \mathrm{superna-}$ tants over the column. Protein was eluted using the same buffer but with $250 \mathrm{mM}$ imidazole. Fc-containing proteins were captured on a protein A-containing MabSelect Sure affinity resin (GE Healthcare) conditioned using phosphate buffered saline (PBS). Protein was eluted using 0.1 $\mathrm{M}$ glycine, $\mathrm{pH} 3.0$ and neutralized using $1 \mathrm{M}$ Tris $\mathrm{pH}$ 8.5. If proteins were $>90 \%$ pure by analytical size exclusion chromatography (SEC) after affinity capture, they were buffer exchanged into PBS at $\mathrm{pH} 7.2$ using VIVASPIN six concentrators with a $10 \mathrm{kDa}$ MW cut-off; otherwise, they were passed over a preparative SEC column (Zepax SRT10C SEC3000).

Analytical characterization of the purity of the proteins was performed using an Agilent 1100 HPLC equipped with a Phenomenex Yarra SEC-3000 $(300 \times 7.8 \mathrm{~cm})$ column using a $100 \mathrm{mM}$ phosphate, $150 \mathrm{mM} \mathrm{NaCl}, 0.05 \%$ azide, pH 6.8 running buffer. Sodium dodecyl sulfate-polyacrylamide gel electrophoresis (SDS-PAGE) was performed using $4 \%-12 \%$ Bis-Tris gels according to the manufacturer (Life Technologies). Reductions and alkylations were performed with $1 \mathrm{mM}$ dithiothreitol followed by $1 \mathrm{mM}$ N-ethylmaleimide at the heating step.

\section{rTCR/anti-CD3 BFP competition flow cytometry assay}

The anti-CD3 flow cytometry competition assay was performed essentially as described previously ${ }^{11}$ with a few minor modifications throughout the protocol. Jurkat tumor cells (ATCC, cat\#TIB-152) grew in suspension in Roswell Park Memorial Institute (RPMI) 1640 media $/ 10 \%$ fetal bovine serum (FBS) Corning/gentamicin (Gibco) at $37^{\circ} \mathrm{C}, 5 \% \mathrm{CO}_{2}$. Cells were transferred to centrifuge tubes and pelleted by centrifugation at $170 \mathrm{~g}$ for $7 \mathrm{~min}$. Cells were washed once with PBS buffer. All subsequent steps were performed on ice. Henceforth, 'wash buffer' was PBS/2\% FBS $/ 0.05 \% \mathrm{NaN}_{3} / 10 \%$ normal goat serum with extra $10 \%$ FBS. Blocking buffer was wash buffer supplemented with human BD Fc Bbock (BD Biosciences, cat\#564220). The cells were resuspended in blocking buffer for $15 \mathrm{~min}$, pelleted, washed $2 \times$, and resuspended in wash buffer before adding $50 \mu \mathrm{L}$ of the cells $\left(0.5 \times 10^{6}\right.$ cells/well) to 96 -well plates (Corning 3799). Next cells were pelleted by centrifugation at $170 \mathrm{~g}$ for $7 \mathrm{~min}$ and wash buffer was aspirated off each well. In a separate 96-well plate (Corning 3879), the rTCR/ anti-CD3 BFPs and control mAbs were added to the wells starting at $1000 \mathrm{nM}$ and titrated using threefold dilutions (working concentrations were $2 \times$ ). rTCR/anti-CD3 $\mathrm{BFP}$ and $\mathrm{mAb}$ dilutions were mixed with equal volume of phycoerythrin (PE)-SP34-2 (final concentration of $2.25 \mu \mathrm{g} / \mathrm{mL}$, BD Biosciences cat\#552127). This sample mixture was transferred to the 96-well plate (Corning 3799 ) with cells at $100 \mu \mathrm{L} /$ well and incubated $45 \mathrm{~min}$. The cells were pelleted and washed three times. Finally, the cells were resuspended in wash buffer with PI (Molecular Probes cat\#P3566) and covered with foil. The cells were then acquired on a Becton Dickinson LSRFortessa flow cytometer with BD FACSDiva software V.8.0.1, and data were analyzed using FlowJo V.10.7.1. The gating strategies for distinguishing live versus dead cells and single cells versus doublets/multiplets that were used for Jurkat cells are provided in online suppplemental figures S2 and S3.

\section{MHC-peptide tumor cell binding of rTCR/anti-CD3 BFPs}

Cell surface MHC-peptide binding of the BFPs was performed essentially as described previously ${ }^{11}$ with minor modifications throughout the protocol. Cell culture was performed as described previously. The day before running flow cytometry, T25 flasks were seeded with Saos-2 (ATCC cat\#HTB-85) or HCT116 (ATCC cat\#CCL-247) cells in 'growth buffer' containing RPMI 1640/10\% FBS (Corning), gentamicin (Gibco) at $37^{\circ} \mathrm{C}, 5 \% \mathrm{CO}_{2}$. The next morning, Saos-2 and HCT-116 tumor cells were washed once with growth buffer. NY-ES0-1 peptide SLLMWITQC (CPC Scientific, product\#824724) was added to Saos-2 cells and MAGE-A3 Peptide: EVDPIGHLY (GenScript, product\#SC1848) was added to the HCT-116 tumor cells (or not as a negative control) at $6 \mu \mathrm{g} / \mathrm{mL}$ for 3 hours at $37{ }^{\circ} \mathrm{C}, 5 \% \mathrm{CO}_{2}$. Next, excess peptide was aspirated off and the cells were washed $3 \times$ with PBS buffer. The tumor cells were lifted from the T25 flasks using Accutase (Innovative Technologies cat\#AT104). All subsequent steps were performed on ice. Henceforth, wash buffer was PBS/2\% FBS/0.05\% sodium azide $/ 10 \%$ normal goat serum with extra 10\% FBS. 'Blocking buffer' was wash buffer supplemented with Human BD Fc Block (BD Biosciences cat\#564220). The cells were resuspended in blocking buffer for $15 \mathrm{~min}$, pelleted, washed $3 \times$, and resuspended in wash buffer before adding $50 \mu \mathrm{L}$ of the cells $\left(0.2 \times 10^{6}\right.$ cells/well for tumor cells) to 96-well plates (Corning 3799). For the tumor cells, the rTCR/anti-CD3 BFPs and recombinantly produced anti-CD3 chimeric SP34 hIgG1_N297 $\mathrm{Q}^{16}$ were added to the wells at 30.0, 3.0, 0.3 , and $0.03 \mu \mathrm{g} / \mathrm{mL}$ and incubated $45 \mathrm{~min}$. The cells were pelleted and washed, and the supernatants were aspirated again before adding $100 \mu \mathrm{L}$ R-PE-conjugated goat anti-human lambda (1:500 dilution; Southern Biotechnology cat\#2070-09) or PE-mouse anti-Histag antibody (1:20 dilution, BioLegend cat\#362603) in wash buffer for $45 \mathrm{~min}$. The cells were pelleted and washed again. Finally, the cells were resuspended in wash buffer with 1:1000 propidium iodide (PI, Molecular Probes cat\#P3566) and covered with foil. The cells were then acquired on a Becton Dickinson LSRFortessa flow cytometer with BD FACSDiva software V.8.0.1, and data were analyzed using FlowJo V.10.7.1. The gating strategies for distinguishing live versus dead cells and single cells versus doublets/multiplets that were used for Saos-2 are shown in online supplemental figures S4 and S5, respectively, and virtually identical to the gating strategies for HCT-116 cells. 
T cell-redirected lysis activity of the rTCR/anti-CD3 BFPs using exogenous MAGE-A3 or NY-ESO-1 peptide

Redirected lysis assays using exogenous cancer testis antigen peptides were performed essentially as described previously ${ }^{11}$ with minor modifications throughout the protocol. Saos-2 and HCT-116 cells were cultured as described earlier. Primary naïve T cells were from StemExpress (cat\#PB03020C, Donor\#D001003581) or AllCells (cat\# PB009-1F, Lt\# 3009286). For the assay, tumor cells were removed from their culture flasks using Accutase as describe previously. The tumor cells were resuspended in complete media and seeded at 5000 cells/well in 96-well black, clear bottom plates (PerkinElmer) overnight at 5\% $\mathrm{CO}_{2}$ and $37^{\circ} \mathrm{C}$. The next day, the media were removed and SLLMWITQC (NY-ESO-1) peptide or HCT-116 used EVDPIGHLY (MAGE-A3) peptide was added to Saos-2 or HCT-116 cells, respectively, $100 \mu \mathrm{L}$ total volume, for 3 hours. Next, $50 \mu \mathrm{L}$ of the peptide solution was removed from each well and rTCR/anti-CD3 BFPs were added $(50 \mu \mathrm{L} /$ well at $4 \times$ ) or control antibodies (chimeric SP34 IgG1-N297Q antiCD3 and irrelevant IgG1) were then titrated onto the cells using $1: 10$ serial dilutions starting at $200 \mathrm{nM}(2 \times)$ and ending at $0.002 \mathrm{nM}(2 \times)$ in triplicate for $30 \mathrm{~min}$. During this period, primary $\mathrm{T}$ cells were thawed and washed twice in complete media and gentamicin. T cells $(100 \mu \mathrm{L})$ were then added at $50 \mathrm{~K}$ cells/well ( $200 \mu \mathrm{L}$ total volume). After the T cells were added, the rTCR/anti-CD3 BFPs were $1 \times$ (starting at $100 \mathrm{nM}$ and ending at $0.001 \mathrm{nM}$ ) and the peptide was at $1.5 \mu \mathrm{g} / \mathrm{mL}$ for the duration of the experiment. Non-peptide plates were treated the same as those with peptide except for the absence of peptide in the procedure. Cells were incubated for 48 hours at $5 \% \mathrm{CO}_{2}$ and $37^{\circ} \mathrm{C}$. At 48 hours, the plates were washed gently (twice) with serum free RPMI 1640. Finally, to determine the amount of tumor cells alive at the end of the incubations, $100 \mu \mathrm{L}$ RPMI 1640 and $100 \mu \mathrm{L}$ Cell Titer Glo reagent (Promega) were added, mixed for $2 \mathrm{~min}$ on a shaker (slowly) and incubated for $10 \mathrm{~min}$ in the dark. Lastly, the luminescence was read on an EnVision V.2130 multilabel reader.

\section{T cell-redirected lysis activity of the rTCR/anti-CD3 BFPs using endogenous levels of MAGE-A3 or NY-ESO-1 peptide}

Redirected lysis assays using endogenous levels of cancer testis antigen were performed essentially as described previously $^{11}$ with minor modifications throughout the protocols. Primary T cells (StemCell cat\#70024, lot \# 200172003C, Donor RG1010) were thawed and resuspended in complete media (RPMI 1640/10\% FBS Corning/gentamicin, Gibco) containing anti-CD28 (BD Biosciences 555725, CD28.27, $2.5 \mu \mathrm{g} / \mathrm{mL}$ ) and interleukin-2 (R\&D systems 202-IL/CF, $2 \mathrm{ng} / \mathrm{mL}$ ) and expanded by being placed in flasks precoated overnight with antiCD3 (BD Biosciences cat\#555329, UCHT1, $5 \mu \mathrm{g} / \mathrm{mL}$ ). T cell expansion was allowed to proceed until day 4 . On day 3 , the tumor cells were resuspended in complete media and seeded at 5000 cells/well in 96-well black, clear bottom plates (PerkinElmer) overnight at $5 \% \mathrm{CO}_{2}$ and $37^{\circ} \mathrm{C}$. On day $4,50 \mathrm{~K}$ expanded $\mathrm{T}$ cells/well were washed
$2 \times$ in complete media and added to each well with HCT116 and A375 tumor cells. Prior to the addition of the T cells, media was removed and the rTCR/anti-CD3 BFPs were titrated into the plates with tumor cells starting at a final concentration of $200 \mathrm{nM}$ with 1:10 serial dilutions ending at 14:00 $(0.002 \mathrm{nM})$. The $\mathrm{T}$ cell/tumor cell/BFP (or MAb) mixtures were allowed to incubate for 24 hours, then the plates were washed gently (twice) with serum free RPMI 1640. Finally, to determine the amount of tumor cells alive at the end of the incubations, $100 \mu \mathrm{L}$ RPMI 1640 and $100 \mu \mathrm{L}$ Cell Titer Glo reagent (Promega) were added, mixed for 2 min on a shaker (slowly) and incubated for $10 \mathrm{~min}$ in the dark. Lastly, the luminescence was read on an EnVision V.2130 multilabel reader.

\section{RESULTS \\ Expression and characterization of TCR-SCFv, TCR-Fab, and TCR-IgG BFPs}

Two well-characterized rTCRs were chosen for the study of various rTCR/anti-CD3 BFP geometries. The first was 1G4_113, an affinity-matured clone of the 1G4 anti-NYESO-1/HLA-A2 TCR, ${ }^{17}$ and the second was an affinitymatured anti-MAGE-A3/HLA-A1 TCR. ${ }^{14}$ Initially, three rTCR/anti-CD3 geometries were assessed: TCR-scFv, TCR-Fab, and TCR-IgG (figure 1A). The anti-CD3 moiety was SP34 constructed as a VH(GGGGS) ${ }_{4} \mathrm{VL}(\mathrm{scFv})$, a chimeric mouse Fv fused to human $\mathrm{CH} 1 / \mathrm{C} \lambda(\mathrm{Fab})$, or an effector-less IgG1 constant region (IgG1_L234A_ L235A_N297Q) ${ }^{11}$ All TCRs contained stabilized C $\alpha /$ $\mathrm{C} \beta$ designs enabling antibody-like transient expression levels, assembly, and secretion via transient expression in CHO cells. ${ }^{11}$ TCR-IgG heterodimerization was achieved using a previously described IgG-CH3 domain heterodimerization design denoted 7.8.60. ${ }^{11}$

The rTCR/anti-CD3 BFPs expressed and assembled well. Both the NY-ESO-1 and MAGE-A3 BFPs were monodisperse by analytical SEC with the TCR-IgGs, TCR-Fabs, and TCR-scFvs eluting in order based on their molecular weight (figure 1B). By SDS-PAGE, the MAGE-A3directed BFPs displayed the expected molecular weights for full assembly under non-reducing conditions and the expected individual chains under reducing conditions (figure 1C). SDS-PAGE analysis of the 1G4_113 NY-ESO1-directed TCR-Fab and TCR-scFv looked similar to their MAGE-A3-directed counterparts (online supplemental figure $\mathrm{S} 1$ )

\section{Functional binding of rTCR/anti-CD3 BFPs}

The ability of the rTCR/anti-CD3 BFPs to bind their target antigens was assessed using flow cytometry. First, binding to CD3 antigen was assessed using the Jurkat $\mathrm{T}$ cell leukemia line. All the BFPs bound Jurkat cells (online supplemental figures S2 and S3). We found the most quantitative method for comparing the CD3 binding affinity of the BFPs to CD3-expressing cells was to perform a competitive flow cytometry assay that compares the ability of each BFP to compete with a set concentration of PE-conjugated SP34 


\section{A}
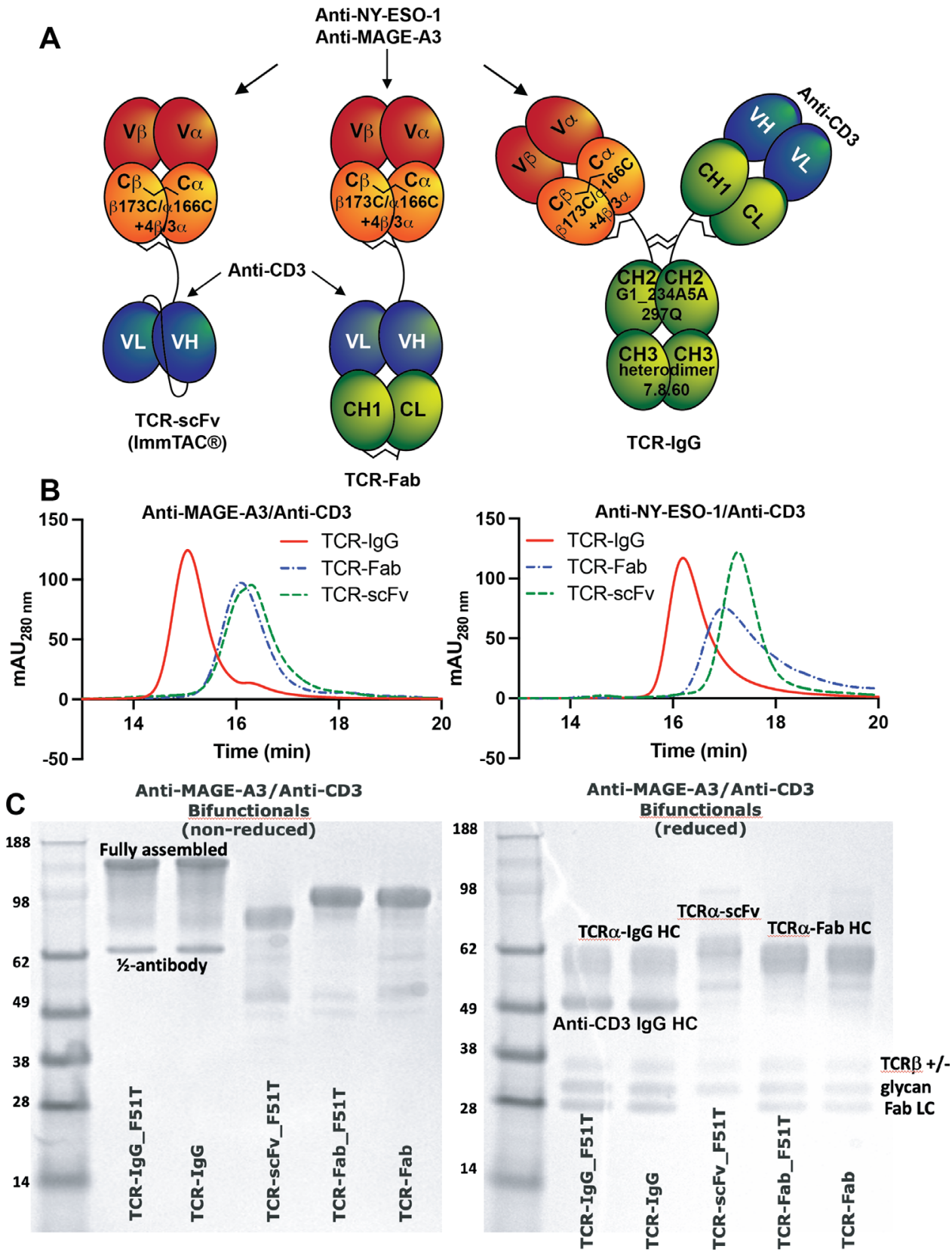

Figure 1 Architecture and biophysical properties of rTCR/anti-CD3 BFPs. (A) Schematic diagrams of the TCR-scFv, TCRFab, and TCR-IgG BFPs. VH and VL are the anti-CD3 antibody heavy and light chain variable domain fragments, respectively. (B) Analytical size exclusion chromatography of the MAGE-A3-directed (left) and the NY-ESO-1-directed (right) rTCR/antiCD3 BFPs directly after protein $\mathrm{A}$ or $\mathrm{Ni}^{2+}{ }^{2+} \mathrm{NTA}$ affinity chromatography. (C) MAGE-A3-directed BFPs analyzed by SDS-PAGE under non-reducing (left) and reducing (right) conditions. BFP, bifunctional protein; rTCR, recombinant T cell receptor-based therapeutic; SDS-PAGE, sodium dodecyl sulfate-polyacrylamide gel electrophoresis; TCR, T cell receptor.

(figure 2 and online supplemental table S1).$^{18}$ Unlabeled, bivalent SP34 was titrated as a control and demonstrated a single-digit nanomolar $\mathrm{IC}_{50}$. Both of the monovalent TCRIgG BFPs demonstrated roughly 10 -fold weaker potency (figure 2 and online supplemental table S1) similar to what has been observed with fully antibody-based monovalent CD3 BsAbs. ${ }^{18}$ Both the TCR-scFv or TCR-Fab BFPs displayed $\sim 10$ fold weaker potency compared with the TCRIgG BFPs likely due to the N-terminal fusion of the TCR hindering the kinetics of CD3 engagement (figure 2 and online supplemental table S1).
Next, binding of the BFP rTCR moieties to cell lines displaying specific MHC/peptide complexes was assessed. NY-ESO-1 binding was performed on HLA-A2 ${ }^{\mathrm{High}}$ Saos-2 osteosarcoma cells preloaded with the NY-ESO-1 ${ }_{157-165}$ SLLMWITQC peptide. All three NY-ESO-1-directed BFPs bound the Saos-2 cell line with roughly similar $\mathrm{EC}_{50} \mathrm{~s}$ though the TCR-IgG BFP did not saturate at the same level as the TCR-Fab and its $\mathrm{EC}_{50}$ was slightly weaker (online supplemental figures S4 and S5, figure 2, online supplemental table S1). All three MAGE-A3-directed BFPs bound HLA-A1 ${ }^{\text {High }}$ HCT116 colon carcinoma cells 

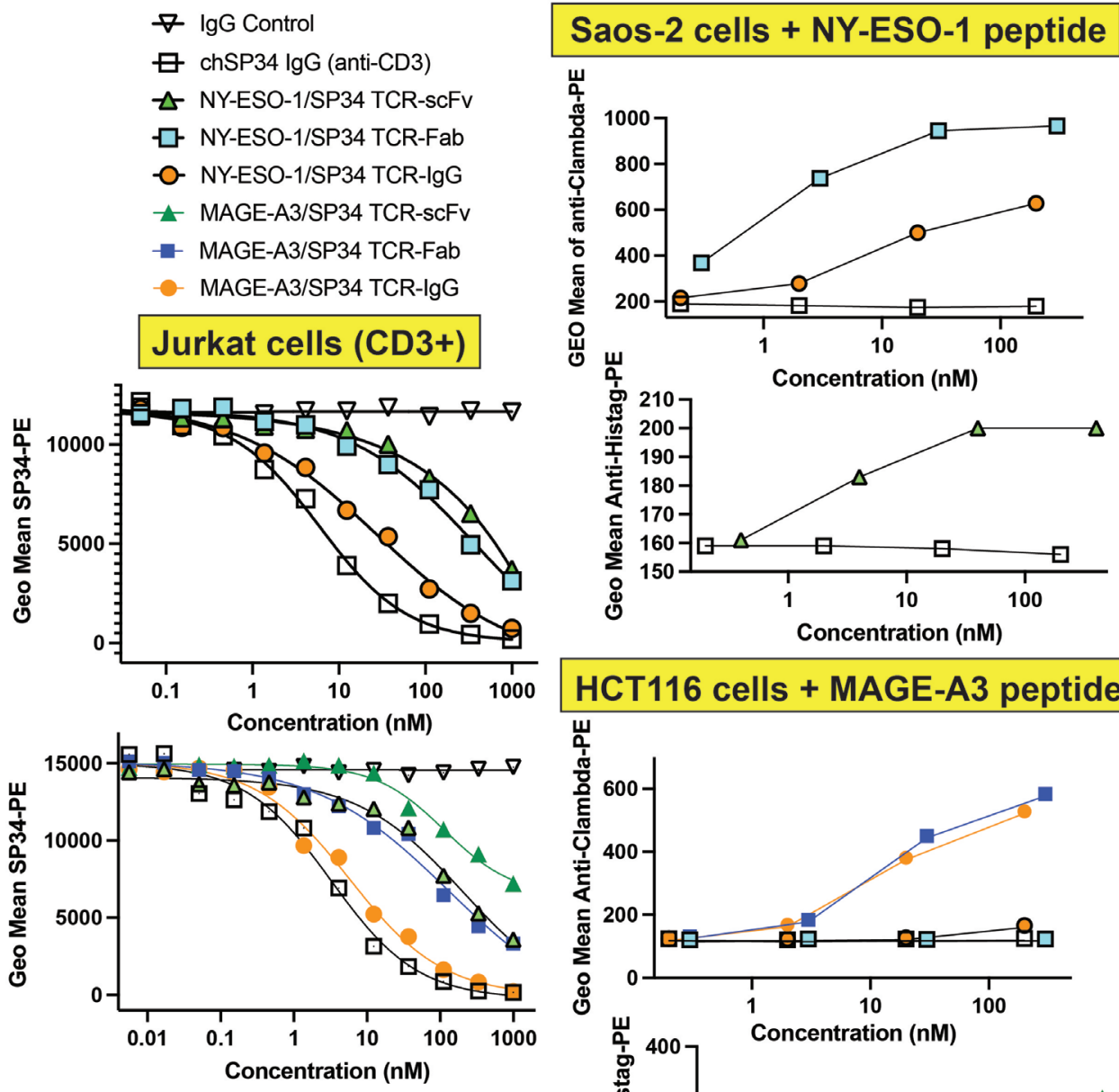

Figure 2 Cell-binding capability of rTCR/anti-CD3 BFPs to both CD3-expressing Jurkat cells (left), NY-ESO-1 ${ }_{157-165}$ SLLMWITQC-labeled SaOS-2 (NY-ESO-1) cells, or MAGE-A3 ${ }_{168-176}$ EVDPIGHLY-labeled HCT116 cells. Jurkat cell potency was assessed by competition with anti-CD3 SP34-PE, while SaOS-2 and HCT116 binding potency was assessed using an antihuman lambda-PE secondary (TCR-IgG and TCR-Fab) or anti-Histag-PE secondary (TCR-scFv). BFP, bifunctional protein; PE, phycoerythrin; rTCR, recombinant T cell receptor-based therapeutic; TCR, T cell receptor.

preloaded with the MAGE-A3 ${ }_{168-176}$ EVDPIGHLY peptide with near identical $\mathrm{EC}_{50} \mathrm{~s}$ (figure 2, online supplemental table S1). No non-specific binding of NY-ESO-1-directed BFPs to the HCT116 line preloaded with the MAGE-A3 peptide occurred, highlighting the HLA/peptide specificity of the BFPs (figure 2). An affinity matured version of the MAGE-A3-directed TCR-IgG and TCR-Fabs (F51T) ${ }^{14}$ demonstrated only a 2-fold $\mathrm{EC}_{50}$ improvement; therefore, we focused subsequent efforts on the original MAGE-A3 TCR-directed BFPs for functional analyses. Overall, both sets of rTCR/anti-CD3 BFPs demonstrated strong cell surface $\mathrm{MHC} /$ peptide and $\mathrm{CD} 3 \varepsilon$ binding capabilities (online supplemental table S1).

\section{T cell-engaging activity of rTCR/anti-CD3 BFPs}

The BFPs were assessed for their ability to redirect $\mathrm{T}$ cells to kill tumor cells displaying the correct HLA-peptide complex. First, the BFPs directed toward MAGE-A3 were mixed with MAGE-A3-peptide-loaded HCT116 cells and naive (rested/non-activated) T cells. The TCR-Fab and TCR-scFv were able to stimulate and redirect the T cells to kill HCT116 in a dose-dependent manner (figure 3A), while the TCR-IgG was inactive. Interestingly, the potency of the TCR-Fab was significantly higher than that of the TCR-scFv. The NY-ESO-1-directed BFPs had no activity against HCT116 cells preloaded with MAGE-A3 peptide. When applied to HLA-A2 ${ }^{\text {High }}$ Saos-2 cells preloaded with NY-ESO-1 peptide, the NY-ESO-1 TCR-Fab and TCR-scFv BFPs could stimulate and redirect $\mathrm{T}$ cells to kill the Saos-2 cells (figure 3B). As with the MAGE-A3-directed TCR-IgG, the NY-ESO-1 TCR-IgG was inactive. Overall, both BFP datasets demonstrate that the TCR-Fab and TCR-scFv moieties can stimulate and redirect $\mathrm{T}$ cells towards tumor cells 


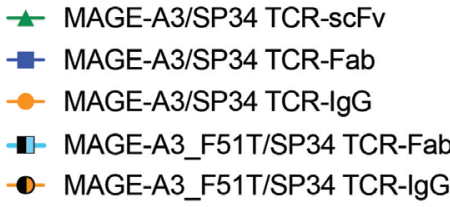

$$
\begin{aligned}
& \text { \# IgG Control } \\
& \theta \text { chSP34 IgG } \\
& \triangle \text { NY-ESO-1/SP34 TCR-scFv } \\
& \square \text { NY-ESO-1/SP34 TCR-Fab } \\
& \text { ○- NY-ESO-1/SP34 TCR-IgG }
\end{aligned}
$$
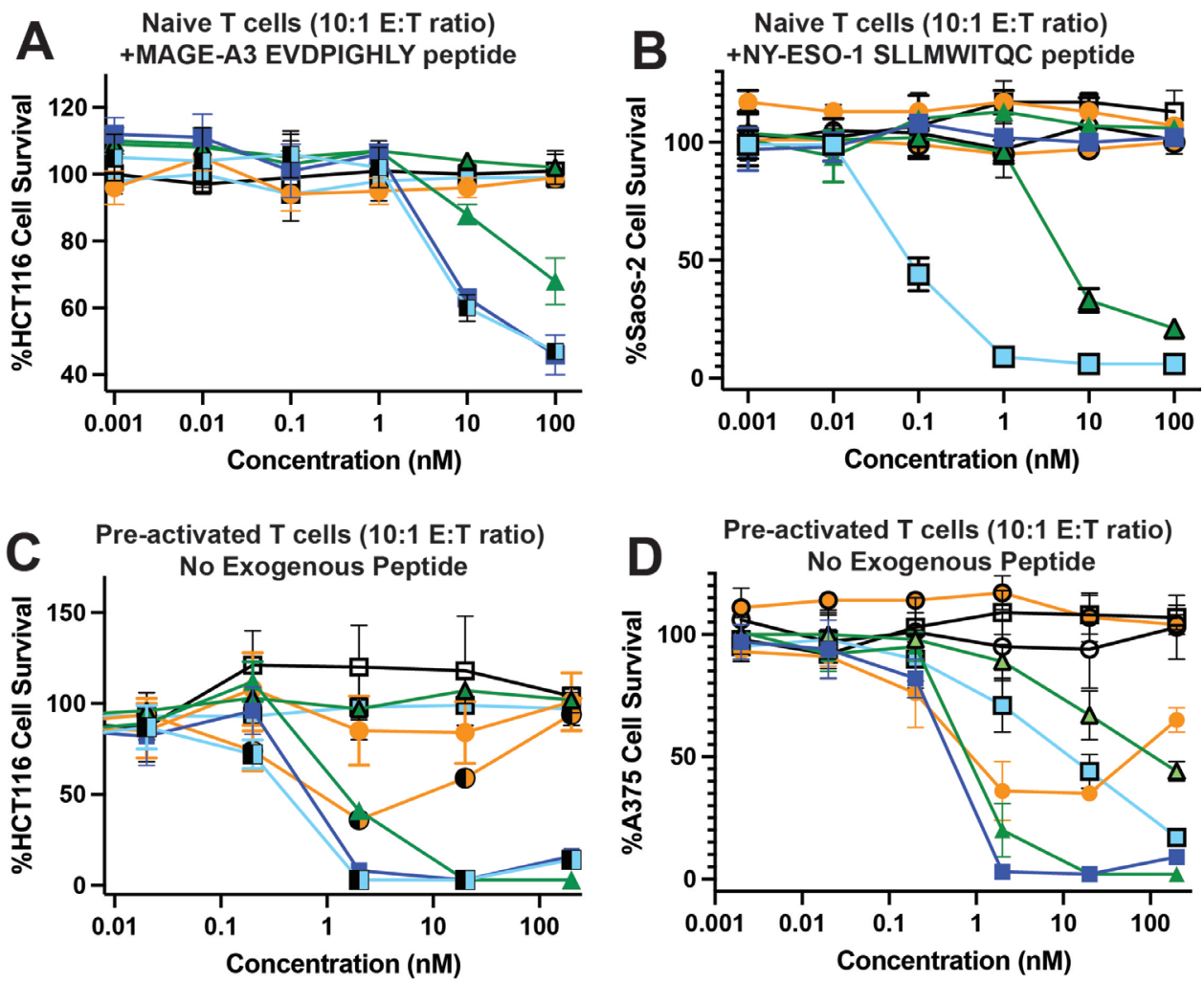

Figure 3 Comparative T cell-redirected killing of HLA/peptide ${ }^{+}$tumorcell lines via the rTCR/anti-CD3 BFPs. BFP-induced redirection of naïve T cells to kill HLA-A1 ${ }^{+} \mathrm{HCT} 116$ colorectal cancer cells $(\mathrm{A})$ or HLA-A2 ${ }^{+}$Saos-2 osteosarcoma cells (B) preincubated with MAGE-A3 ${ }_{168-176}$ EVDPIGHLY or NY-ESO-1 ${ }_{157-165}$ SLLMWITQC peptides, respectively. BFP-induced killing of $\mathrm{HLA}-\mathrm{A} 1^{+} / \mathrm{MAGE}-\mathrm{A} 3^{+} / \mathrm{HLA}-\mathrm{A} 2^{+} / \mathrm{NY}-\mathrm{ESO}-1^{-} \mathrm{HCT} 116$ cells $(\mathrm{C})$ or HLA-A $1^{+} / \mathrm{MAGE}^{-\mathrm{A}} 3^{+} / \mathrm{HLA}-\mathrm{A} 2^{+} / \mathrm{NY}-\mathrm{ESO}-1^{+}$A375 malignant melanoma cells (D) using preactivated T cells. BFP, bifunctional protein; E:T, effector cell to tumor cell ratio; rTCR, recombinant T cell receptor-based therapeutic; TCR, T cell receptor.

displaying their target antigens, while the TCR-IgG format is either inactive or much less active. In both cases, the TCRFab format was more potent than the TCR-scFv format.

Next, we evaluated the ability of the MAGE-A3 and NY-ESO-1 BFPs to redirect $\mathrm{T}$ cells to kill tumor cells expressing endogenous levels of antigen/HLA. The first cancer cell line evaluated was HCT116, which endogenously expresses the MAGE-A3/HLA-A1, but does not express NY-ESO-1. ${ }^{19} 20$ The MAGE-A3-directed TCR-Fab and TCR-scFv could induce $\mathrm{T}$ cell-mediated killing of HCT116 tumor cells while the NY-ESO-1-directed BFPs had no activity. Again, the TCR-Fab was more potent than the TCR-scFv. The MAGE-A3-directed TCR-IgG was inactive; however, the higher affinity F51T TCR-IgG BFP variant ${ }^{14}$ displayed activity that diminished in a bellshaped profile at higher concentrations (figure 3C). When the MAGE-A3- and NY-ESO-1-directed BFPs were applied to A375 cells, which endogenously express both MAGE-A3/HLA-A1 and NY-ESO-1/HLA-A2, both sets of TCR-Fabs and TCR-scFvs induced activated T cells to kill the A375 cells (figure 3D). Overall, the MAGE-A3 BFPs more potently induced T-cell killing of the A375 cells compared with the NY-ESO-1 BFPs (figure 3D). Again, the TCR-Fabs were slightly more potent than the TCRscFvs (figure 3D). The MAGE-A3 TCR-IgG did redirect T cells with high potency to kill A375 cells, but killing only reached $60 \%$ and demonstrated a bell-shaped activity curve similar to the data observed for the F51T MAGE-A3 TCR-IgG on HCT116 cells pre-loaded with the MAGE-A3 peptide (figure 3C,D).

\section{Expanded rTCR/CD3 BFP geometry/valency evaluation}

The scope of rTCR/anti-CD3 BFP analyses was expanded to include the impact of rTCR affinity and additional BFP geometries. The geometries chosen for investigation were inspired by the most prevalent $\mathrm{T}$ cell-engaging $\mathrm{BsAb}$ geometries, ${ }^{10}$ and our observation that $\mathrm{T}$ cell-engaging BsAbs with tandem formats comprising an N-terminal TSA binding domain followed by an anti-CD3 domain showed superior potency over IgG-format bifunctionals in many of our assays. ${ }^{16} 18$ Also, many $\mathrm{T}$ cell-engaging BsAbs are moving toward 2:1 formats (2 TSA moieties: 
$1 \mathrm{CD} 3$ moiety) that use modest affinity and avidity to achieve improved specific binding to tumor cells overexpressing TSA and to avoid normal tissues expressing lower TSA levels. ${ }^{10}$ Lastly, formats with an antibody-Fc to impart antibody-like pharmacokinetics were evaluated. Given the generally similar results obtained comparing the TCR-Fab, TCR-scFv, and TCR-IgG formats directed to MAGE-A3/HLA-A1 and NY-ESO-1/HLA-A2, we decided to focus on the NY-ESO-1 BFPs allowing the construction and testing of more formats.

We first investigated the requirement for high affinity. Previously, we found that rTCR/anti-CD3 BFPs with TCR affinities at or below $1 \mathrm{nM}$ did not show significant potency differences in vitro. ${ }^{11}$ Therefore, we investigated the impact of reducing the affinity by one or two orders of magnitude. $\mathrm{Li}$ and coworkers published a study that included anti-NY-ESO-1 rTCR sequences ranging from low pM to $\mu \mathrm{M} \mathrm{K} \mathrm{K}_{\mathrm{D}}$ for HLA-A2/peptide. ${ }^{17}$ TCR-Fab BFPs with HLA-A2/NY-ESO-1 peptide binding affinities ranging from $1 \mathrm{nM}$ to $200 \mathrm{nM}$ were generated (1G4_122 1 nM, $1 \mathrm{G} 4 \_006 \sim 10 \mathrm{nM}$, and $1 \mathrm{G} 4 \_033 \sim 200 \mathrm{nM}^{17}$ and tested for redirected lysis activity on Saos-2 cells loaded with NY-ESO-1 peptide. While the $1 \mathrm{nM}$ TCR-Fab demonstrated redirected lysis potency similar to the $25 \mathrm{pM}$ TCR-Fab, both the $10 \mathrm{nM}$ and $200 \mathrm{nM} \mathrm{K}_{\mathrm{D}}$ TCR-Fab BFPs had highly attenuated activity and potency ( $>2$ orders of magnitude, figure 4A), suggesting high HLA/peptide affinity is crucial for the activity and potency of these molecules in agreement with what has been described previously. ${ }^{7}$

Next, the geometric aspects of the rTCR/anti-CD3 moieties were evaluated in more depth. First, we evaluated modifications of the original TCR-IgG and TCRFab BFPs (using the 1G4_122 anti-NY-ESO-1 TCR with a $1 \mathrm{nM} \mathrm{K}_{\mathrm{D}}$ ) to assess whether moving to the tandem format (TCR-Fab-Fc) or a 2:1 tandem format (TCR-Fab-IgG) would improve the potency of an Fc-containing BFP. ${ }^{17}$ The tandem format, TCR-Fab-Fc, did lead to a modest increase in potency over the TCR-IgG format, though still two orders of magnitude weaker than the tandem TCR-Fab format lacking an IgG-Fc (figure 4A). Diversifying the linker length from a (GGGGS) ${ }_{3}$ to (GGGGS) or (GGGGS) 5 linker did not impact these results. Adding an additional rTCR arm within the TCR-Fab-IgG format eradicated all activity.

Subsequently, we evaluated multiple 2:1 rTCR:anti-CD3 tandem formats. Adding a second rTCR to the N-terminus of the TCR-Fab (denoted 2TCR.1-Fab) did not improve potency over the original TCR-Fab format and appeared to induce an activity loss at higher BFP concentrations similar to what was observed for the MAGE-A3 TCR-IgG BFP (figure 4B). Moving the anti-CD3 Fab moiety to the central or N-terminal positions (denoted 2TCR.2-Fab and 2TCR.3-Fab, respectively) each led to weaker potency and activity with the N-terminal anti-CD3 Fab format having no activity (figure 4B). Lastly, we evaluated additional IgG-Fc containing 2:1 formats (denoted 2TCR-IgGs). These formats included a variety of contexts investigating the positioning of the second rTCR or the anti-CD3 Fab moieties.
In short, none of these formats were more active than the TCR-IgG format, and most were completely inactive in the assay (figure 4C). Thus, adding a second rTCR to the original constructs did not result in improvements in potency or activity over the original TCR-Fab or TCR-IgG formats and in most cases reduced activity.

\section{DISCUSSION}

Unlike $\mathrm{T}$ cell-engaging BsAbs, the parameters required for potent $\mathrm{rTCR} /$ anti-CD3 $\mathrm{T}$ cell redirection have been poorly characterized. Still, the anti-gp100 rTCR/anti-CD3 ImmTAC is the first T-cell engager to show strong clinical activity in a solid tumor indication, uveal melanoma, in a large phase III trial, ${ }^{12}$ demonstrating the utility of the rTCR-based BFPs and warranting further exploration of rTCR-based T cell engagers. Given the conserved nature of TCR-HLA-peptide interactions, we hypothesized that the parameters dictating activity are likely generalizable, unlike BsAb-based T-cell engagers whose activity depends on both BsAb geometry and the membrane proximity of the targeted epitope. ${ }^{10}$ In agreement with this hypothesis, the impact of BFP geometry on the activity and potency was uniform across both the MAGE-A3/HLAA1- and NY-ESO-1/HLA-A2-directed BFPs. While there is significant diversity in TCR complementarity determining loops and how they engage specific HLA-bound antigenic peptides, the overall angle of approach of the rTCR to its HLA-epitope is a general feature required for the production of a functional immune synapse. ${ }^{21}$ To our surprise, both sets of TCR-Fab BFPs had greater potency than the TCR-scFv format, while the activity of the TCRIgG (antibody-like) format was substantially weaker. We hypothesize that the larger and less flexible TCR-IgG has difficulty forming the tight immune synapse necessary to induce TCR conformational changes, and TCR crosslinking, and possibly the exclusion of large phosphatase receptors as described in the kinetic-segregation model (online supplemental figure S6) ${ }^{22}$ Also, in some circumstances, the TCR-IgG and 2TCR.1-Fab (ie, the fusion of 2 rTCRs and one anti-CD3 Fab) formats demonstrated bell-shaped activity profiles with a loss of activity at higher concentrations. This may result from molecular crowding and expansion of the synapse between $\mathrm{T}$ cell and tumor cell with these larger molecules. All formats containing an IgG-Fc demonstrated poor potency or a total lack of activity. It is possible smaller modifications, for example, acylation or nanobody fusion, could direct these molecules to human serum albumin for improved pharmacokinetics with binding kinetics that allow for controlled release once the molecules enter the immune synapse. The use of diverse anti-CD3 binders with varied epitopes hypothetically could impact the general observations discovered here; however, the epitope space described for anti-CD3 binders is limited and structures determined of different anti-CD3 mAbs bound to CD3 $\varepsilon$ have nearly identical overall epitopes. ${ }^{23} 24$ Thus, it seems unlikely that varying the $\mathrm{CD} 3$ epitope would modify the geometrical 


\section{Controls}
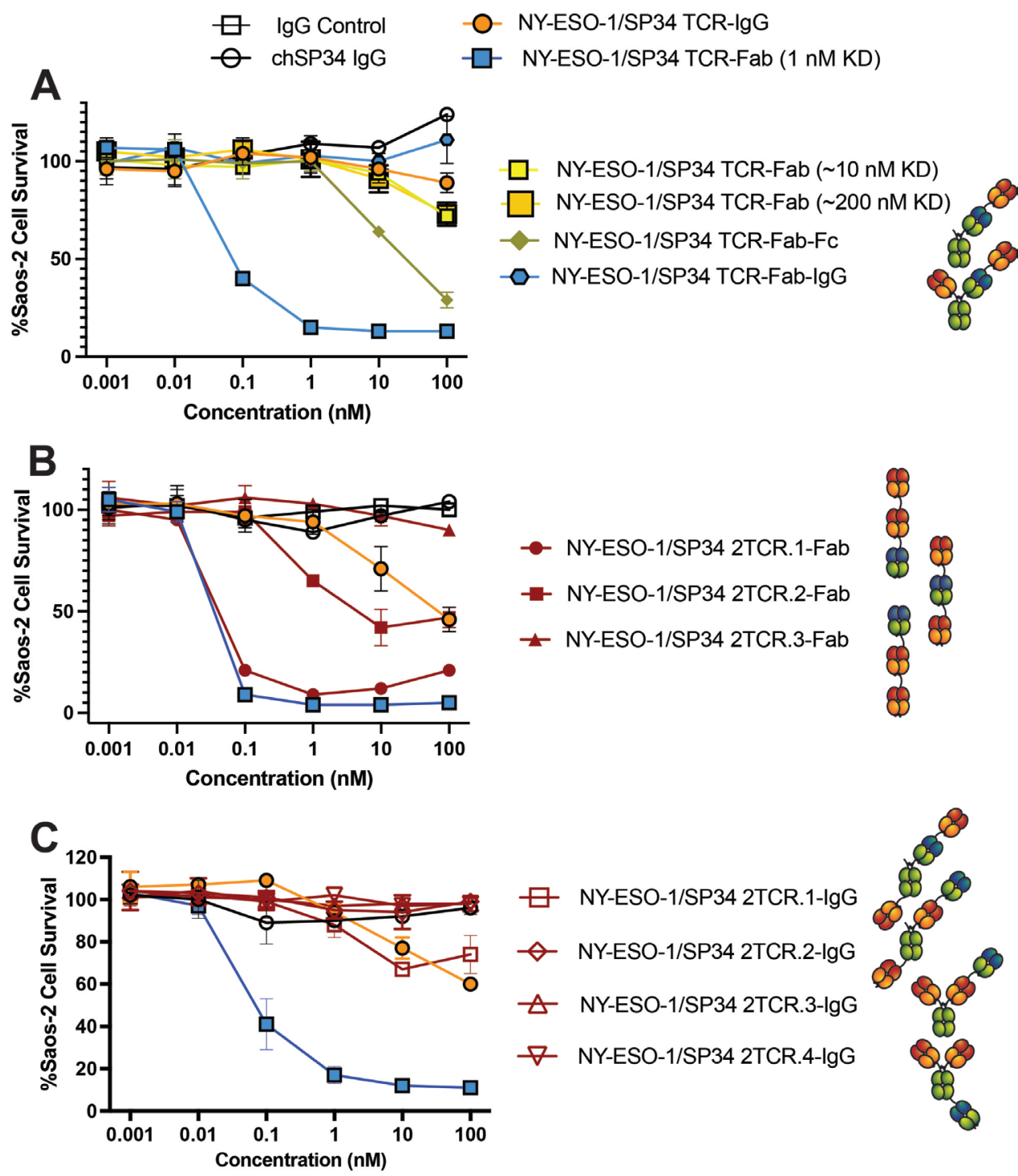

Figure 4 Assessment of affinity and diverse architecture on T cell-redirected lysis activity of NY-ESO-1-directed rTCR/ anti-CD3 BFPs. impact of affinity or addition of an IgG-Fc with or without a second rTCR (A); addition of an additional TCRbinding arm to the tandem format in three separate orientations (B); or complex avid structures containing both an IgG-Fc and a second $r$ TCR in various orientations $(C)$ on the ability of the $r$ TCR/anti-CD3 BFPs to redirect naïve T cells to kill HLA-A2 ${ }^{+}$ SaOS-2 cells labeled with NY-ESO- $1_{157-165}$ SLLMWITQC peptide. As controls, NY-ESO-1 TCR-Fab and TCR-IgG BFPs were used in every experiment. The differences in the redirected lysis potency of the control NY-ESO-1 TCR-Fab and TCR-IgG as well as other rTCR/anti-CD3 BFPs across different experiments can be attributed to the use of different T cell donors. Other than the exceptions described (A), all TCRs had a $1 \mathrm{nM} \mathrm{K}_{\mathrm{D}}$ for the NY-ESO-1/HLA-A2 complex. BFP, bifunctional protein; rTCR, recombinant $\mathrm{T}$ cell receptor-based therapeutic; TCR, T cell receptor.

requirements of rTCR-based BFPs for T-cell redirection uncovered here.

Like BsAb T cell engagers, a complex relationship between HLA/peptide-targeting affinity and potency exists. There appeared to be a threshold near $1 \mathrm{nM}$ for the NY-ESO-1-directed TCR-Fab BFP that enabled potent $\mathrm{T}$ cell redirection that was nearly identical regardless of an affinity difference between $1 \mathrm{G} 4 \_113\left(\mathrm{~K}_{\mathrm{D}} \sim 25 \mathrm{pM}\right)$ and 1G4_122 $\left(\mathrm{K}_{\mathrm{D}} \sim 1 \mathrm{nM}\right)^{11}$ but which was rapidly degraded by reducing affinity further. Such a result is likely related to the avidity of molecular engagement at the immune synapse. Recently, a diabody-based TCR-mimic BsAb $(\sim 50 \mathrm{kDa})$ was shown to have antitumor activity against a common mutant of the tumor suppressor p53 protein, mutants of oncogenic Ras enzyme, and other oncogenes. ${ }^{25}$ The diabody directed to mutant TP53 had an affinity of $86 \mathrm{nM}$ and was still capable of potent $\mathrm{T}$ cell redirection. ${ }^{25}$ One possibility for this potency discrepancy is the known high potency of the UCHT1 anti-CD3 with stable binding to the $\mathrm{T}$ cell surface, allowing high avidity display of the anti-TP53 portion of their molecule. ${ }^{26}$ High-affinity binding to CD3e, however, is likely to result in general $\mathrm{T}$ cell activation, and numerous studies have shown that low-affinity CD3e binding may facilitate less 
systemic cytokine release and improved targeting of these compounds to the tissue sites harboring the epitopes of interest. $^{182728}$

We show that the TCR-Fab format had the best potency. This format has not been widely adopted likely because it is less amenable to production in bacterial systems, which has been the standard for rTCR molecules. An intrinsically unfolded $\mathrm{CH} 1$ domain ${ }^{29}$ and likely issues with three chain oxidative folding would likely preclude bacterial production of a TCR-Fab. Soluble TCR production in mammalian cells has also been fraught with secretion and assembly issues and strong glycosylation, which can result in binding to lectin-containing receptors such as the asialoglycan receptor in the liver or DC-SIGN on dendritic cells impacting their pharmacokinetics, trafficking, and immunogenicity. ${ }^{30}$ However, design of intrinsically stable rTCRs, as described recently, ${ }^{11}$ resulted in significant decreases in glycosylation with good assembly and expression from mammalian cells. Thus, expression of a stabilized TCR-Fab format using mammalian expression systems in the future may significantly improve both the function and production of rTCR/anti-CD3 BFPs for therapeutic use.

Present affiliations The present affiliation of Stephen J Demarest is: Tentarix Biotherapeutics, California, San Diego, USA.

Contributors KJF, AS, and SJD conceived the project. KJF, AS, and FH performed the experiments. KJF, AS, and SJD wrote and edited the manuscript.

Funding The authors have not declared a specific grant for this research from any funding agency in the public, commercial or not-for-profit sectors.

Competing interests All authors are or were employees of Eli Lilly and Company.

Patient consent for publication Not applicable.

Provenance and peer review Not commissioned; externally peer reviewed.

Data availability statement All data relevant to the study are included in the article or uploaded as supplementary information.

Supplemental material This content has been supplied by the author(s). It has not been vetted by BMJ Publishing Group Limited (BMJ) and may not have been peer-reviewed. Any opinions or recommendations discussed are solely those of the author(s) and are not endorsed by BMJ. BMJ disclaims all liability and responsibility arising from any reliance placed on the content. Where the content includes any translated material, BMJ does not warrant the accuracy and reliability of the translations (including but not limited to local regulations, clinical guidelines, terminology, drug names and drug dosages), and is not responsible for any error and/or omissions arising from translation and adaptation or otherwise.

Open access This is an open access article distributed in accordance with the Creative Commons Attribution Non Commercial (CC BY-NC 4.0) license, which permits others to distribute, remix, adapt, build upon this work non-commercially, and license their derivative works on different terms, provided the original work is properly cited, appropriate credit is given, any changes made indicated, and the use is non-commercial. See http://creativecommons.org/licenses/by-nc/4.0/.

\section{ORCID iD}

Stephen J Demarest http://orcid.org/0000-0001-7353-4044

\section{REFERENCES}

1 Pardoll DM. The blockade of immune checkpoints in cancer immunotherapy. Nat Rev Cancer 2012;12:252-64.

2 Goebeler M-E, Bargou RC. T cell-engaging therapies - BiTEs and beyond. Nat Rev Clin Oncol 2020;17:418-34.

3 June $\mathrm{CH}, \mathrm{O}^{\prime}$ Connor RS, Kawalekar OU, et al. Car T cell immunotherapy for human cancer. Science 2018;359:1361-5.
4 Chandran SS, Klebanoff CA. T cell receptor-based cancer immunotherapy: emerging efficacy and pathways of resistance. Immunol Rev 2019;290:127-47.

5 Joglekar AV, Li G. T cell antigen discovery. Nat Methods 2021:18:873-880.

6 Jones HF, Molvi Z, Klatt MG, et al. Empirical and rational design of $T$ cell receptor-based immunotherapies. Front Immunol 2020;11:585385

7 Lowe KL, Cole D, Kenefeck R, et al. Novel TCR-based biologics: mobilising T cells to warm 'cold' tumours. Cancer Treat Rev 2019;77:35-43

8 Lowe KL, Mackall CL, Norry E, et al. Fludarabine and neurotoxicity in engineered T-cell therapy. Gene Ther 2018;25:176-91.

9 Brandt LJB, Barnkob MB, Michaels YS, et al. Emerging approaches for regulation and control of CAR T cells: a mini review. Front Immunol 2020;11:326.

10 Ellerman D. Bispecific T-cell engagers: towards understanding variables influencing the in vitro potency and tumor selectivity and their modulation to enhance their efficacy and safety. Methods 2019;154:102-17.

11 Froning K, Maguire J, Sereno A, et al. Computational stabilization of $T$ cell receptors allows pairing with antibodies to form bispecifics. Nat Commun 2020;11:2330.

12 Nathan P, Hassel JC, Rutkowski P, et al. Overall survival benefit with Tebentafusp in metastatic uveal melanoma. N Engl J Med 2021;385:1196-206.

13 Gunnarsen KS, Høydahl LS, Neumann RS, et al. Soluble T-cell receptor design influences functional yield in an E. coli chaperoneassisted expression system. PLoS One 2018:13:e0195868.

14 Raman MCC, Rizkallah PJ, Simmons R, et al. Direct molecular mimicry enables off-target cardiovascular toxicity by an enhanced affinity TCR designed for cancer immunotherapy. Sci Rep 2016;6:18851.

15 Rajendra Y, Peery RB, Hougland MD, et al. Transient and stable CHO expression, purification and characterization of novel hetero-dimeric bispecific lgG antibodies. Biotechnol Prog 2017;33:469-77.

16 Wu X, Sereno AJ, Huang F, et al. Fab-based bispecific antibody formats with robust biophysical properties and biological activity. MAbs 2015;7:470-82.

17 Li Y, Moysey R, Molloy PE, et al. Directed evolution of human T-cell receptors with picomolar affinities by phage display. Nat Biotechnol 2005;23:349-54

18 Poussin M, Sereno A, Wu X, et al. Dichotomous impact of affinity on the function of T cell engaging bispecific antibodies. J Immunother Cancer 2021:9:e002444.

19 McCormack E, Adams KJ, Hassan NJ, et al. Bi-specific TCR-anti CD3 redirected T-cell targeting of NY-ESO-1- and LAGE-1-positive tumors. Cancer Immunol Immunother 2013;62:773-85.

20 Sers C, Kuner R, Falk CS, et al. Down-regulation of HLA class I and NKG2D ligands through a concerted action of MAPK and DNA methyltransferases in colorectal cancer cells. Int $J$ Cancer 2009;125:1626-39.

21 Kass I, Buckle AM, Borg NA. Understanding the structural dynamics of TCR-pMHC complex interactions. Trends Immunol 2014;35:604-12.

22 Davis SJ, van der Merwe PA. The kinetic-segregation model: TCR triggering and beyond. Nat Immunol 2006;7:803-9.

23 Kjer-Nielsen L, Dunstone MA, Kostenko L, et al. Crystal structure of the human T cell receptor CD3 epsilon gamma heterodimer complexed to the therapeutic mAb OKT3. Proc Natl Acad Sci U S A 2004:101:7675-80.

24 Arnett KL, Harrison SC, Wiley DC. Crystal structure of a human CD3epsilon/delta dimer in complex with a UCHT1 single-chain antibody fragment. Proc Natl Acad Sci U S A 2004;101:16268-73.

25 Hsiue EH-C, Wright KM, Douglass J, et al. Targeting a neoantigen derived from a common TP53 mutation. Science 2021;371. doi:10.1126/science.abc8697. [Epub ahead of print: 0503 2021].

26 Dopfer EP, Hartl FA, Oberg H-H, et al. The CD3 conformational change in the $\gamma \delta \mathrm{T}$ cell receptor is not triggered by antigens but can be enforced to enhance tumor killing. Cell Rep 2014;7:1704-15.

27 Vafa O, Trinklein ND. Perspective: designing T-cell engagers with better therapeutic windows. Front Oncol 2020;10:446.

28 Mandikian D, Takahashi N, Lo AA, et al. Relative target affinities of T-cell-dependent bispecific antibodies determine biodistribution in a solid tumor mouse model. Mol Cancer Ther 2018;17:776-85.

29 Feige MJ, Groscurth S, Marcinowski M, et al. An unfolded CH1 domain controls the assembly and secretion of IgG antibodies. Mol Cell 2009;34:569-79.

30 Lepenies B, Lee J, Sonkaria S. Targeting C-type lectin receptors with multivalent carbohydrate ligands. Adv Drug Deliv Rev 2013;65:1271-81. 